\title{
Prontuário eletrônico em terapia intensiva: validação de instrumento sobre percepção e satisfação da enfermagem
}

Electronic Medical Records in Intensive Care Units: Validation of an Instrument on Nursing Perception and Satisfaction

Historia clínica electrónica en las unidades de cuidados intensivos: validación del instrumento sobre la percepción y satisfacción del personal de enfermería

Como citar este artigo:

Costa, Daiane Vieira Medeiros; Gomes, Vanessa Rossato; Godoi, Ana Maria Limeira de. Prontuário eletrônico em terapia intensiva: validação de instrumento sobre percepção e satisfação da enfermagem. Revista Cuidarte. 2021;12(2):e1332. http://dx.doi.org/10.15649/cuidarte.1332

Revista Cuidarte

Rev Cuid. May - Ago 2021; 12(2): e1332 doil http://dx.doi.org/10.15649/cuidarte.1332

E-ISSN: 2346-3414

(1) Daiane Vieira Medeiros Costa ${ }^{1}$

(1) Vanessa Rossato Gomes ${ }^{2}$

(1) Ana Maria Limeira de Godoi ${ }^{3}$

1 Escola de Enfermagem de Ribeirão Preto da Universidade de São Paulo. Ribeirão Preto (SP), Brasil. E-mail: daiane.vieira.costa@usp.br

2 Instituto do Coração do Hospital das Clínicas da Faculdade de Medicina da Universidade de São Paulo. São Paulo (SP), Brasil. E-mail: vanrossato@gmail.com

3 Instituto do Coração do Hospital das Clínicas da Faculdade de Medicina da Universidade de São Paulo. São Paulo (SP), Brasil. E-mail: anam.limeiragodoi@gmail.com

\section{Resumo}

Introdução: A assistência à saúde requer o registro das atividades desenvolvidas, realizado por meio do prontuário eletrônico do paciente, ferramenta que permeia muitas tarefas, sobretudo, em unidades de terapia intensiva. Problemas nesses sistemas eletrônicos ocasionam consequências com impacto para a assistência. O objetivo foi avaliar a percepção sobre o uso do prontuário eletrônico e a satisfação dos profissionais de enfermagem intensivistas, bem como validar um instrumento de pesquisa para esse fim. Materiais e Métodos: Estudo transversal, quantitativo, desenvolvido na cidade de São Paulo. O instrumento de coleta foi elaborado pelas autoras e validado com relação à aparência e conteúdo. As variáveis categóricas foram comparadas pelo Teste Exato de Fisher e Qui Quadrado; e as variáveis numéricas pelo Teste de Kruskal-Wallis. Resultados: Participaram do estudo 75 profissionais de enfermagem, a maioria era do sexo feminino, pertencentes a categoria de técnicos de enfermagem. A maioria classificou o prontuário eletrônico do paciente como fácil; referiu conhecimento suficiente sobre o prontuário eletrônico do paciente; relatou quantidade insuficiente de computadores no setor e avaliou melhora na segurança do paciente e da equipe de saúde com a utilização do prontuário eletrônico do paciente. As mulheres reportaram maior insatisfação no uso do prontuário eletrônico do paciente, bem como os técnicos de enfermagem. Os enfermeiros têm maior dificuldade na utilização desta ferramenta. Conclusões: $O$ prontuário eletrônico do paciente é fácil de usar e as principais dificuldades estão relacionadas às funcionalidades, sobretudo para os enfermeiros.

Palavras chave: Tecnologia da Informação; Registros Eletrônicos de Saúde; Informática em Saúde; Cuidados de Enfermagem; Unidades de Terapia Intensiva.

Recebido: junho 25 de 2020

Aceito: fevereiro 2 de 2021

Publicado: maio 18 de 2021 $\square *{ }^{*}$ Correspondencia

Daiane Vieira Medeiros Costa

E-mail:kendy.madero@curnvirtual.edu.co. 


\title{
Electronic Medical Records in Intensive Care Units: Validation of an Instrument on Nursing Perception and Satisfaction
}

\begin{abstract}
Introduction: When providing healthcare, it is necessary to record treatment activities in the patient's electronic medical records, which serves as a tool that impacts several tasks, including those performed in intensive care units. Any failure of these electronic systems has a direct impact on the delivery of healthcare. This study is aimed to assess the perception of the use of electronic medical records and satisfaction levels among intensive care nurses, as well as to evaluate a questionnaire instrument for this purpose. Materials and Methods: A quantitative cross-sectional study was conducted in São Paulo. The instrument used for collection was prepared by the authors, which was also validated in terms of appearance and content. Categorical variables were compared using Fisher's exact test and numerical variables were compared using the Kruskal-Wallis test. Results: 75 nursing professionals, mostly women and classified as nurse technicians, participated in this study. The majority of the participants considered that electronic medical records were easy to use, indicated that they had sufficient knowledge about the management of electronic medical records, noted that the number of computer in the hospital was insufficient, and stated that both patient and healthcare team safety had improved after using the patient's electronic medical records. Male nurses found it more difficult to use this tool. Conclusions: Electronic medical records were found to be easy to use, while the main difficulties are related to their functionality, especially by male nurses.
\end{abstract}

Key words: Information Technology; Eletronic Health Records; Medical Informatics; Nursing Care; Intensive Care Units.

\section{Historia clínica electrónica en las unidades de cuidados intensivos: validación del instrumento sobre la percepción y satisfacción del personal de enfermería}

\section{Resumen}

Introducción: En la asistencia sanitaria es necesario registrar las actividades realizadas a través de la historia clínica electrónica del paciente, la cual se constituye como una herramienta que incide en diversas tareas, entre ellas, las realizadas en las unidades de cuidados intensivos. Cualquier problema que se presente en estos sistemas electrónicos tiene un impacto directo en la prestación de la asistencia sanitaria. El objetivo de este estudio fue evaluar la percepción sobre el uso de la historia clínica electrónica y la satisfacción entre los profesionales de enfermería del área de cuidados intensivos, así como evaluar un instrumento de cuestionario para este fin. Materiales y métodos: Se realizó un estudio transversal cuantitativo en la ciudad de São Paulo. El instrumento de recolección fue elaborado por los autores y validado en cuanto a apariencia y contenido. Las variables categóricas se compararon mediante la prueba exacta de Fisher y la prueba de chi-cuadrado, y las variables numéricas a través de la prueba de Krushal-Wallis. Resultados: En el estudio participaron 75 profesionales de enfermería, la mayoría mujeres, que pertenecían a la categoría de técnicos de enfermería. La mayoría de los participantes consideró que la historia clínica electrónica era fácil de usar, indicó tener conocimiento suficiente sobre el manejo de la historia clínica electrónica, señaló que el número de computadores en el hospital era insuficiente y afirmó que había mejorado la seguridad del paciente y del equipo asistencial con el uso de la historia clínica electrónica del paciente. A los enfermeros les resulta más difícil utilizar esta herramienta. Conclusiones: Se encontró que la historia clínica electrónica resulta fácil de usar, mientras que las principales dificultades están relacionadas con su funcionalidad, especialmente por parte de los enfermeros.

Palabras clave: Tecnología de la Información; Registros Electrónicos de Salud; Informática de la Salud; Atención de Enfermería; Unidades de Cuidados Intensivos. 


\section{Introdução}

O processo de assistência à saúde requer o registro dos atendimentos, procedimentos e atividades prestados pela equipe multiprofissional ao paciente, e consiste em um dever delegado aos profissionais, previsto nos seus respectivos códigos de ética. $\mathrm{O}$ registro sistemático das informações clínicas do indivíduo é realizado por meio do prontuário do paciente,
O registro sistemático das informações clínicas do indivíduo é realizado por meio do prontuário do paciente, documento essencial que proporciona organização da informação e é o principal meio de comunicação entre todos os integrantes da equipe de saúde documento essencial que proporciona organização da informação (clínica e administrativa) e é o principal meio de comunicação entre todos os integrantes da equipe de saúde dentro de uma instituição. Dessa forma, o prontuário garante a continuidade do cuidado e a avaliação da evolução clínica do paciente ${ }^{1,2}$.

O prontuário do paciente é uma fonte de informação primária, indispensável para acompanhar o histórico de saúde e doença do indivíduo, além de gerar conhecimentos no âmbito administrativo, de ensino, pesquisa e aspectos legais, traduzindo-se em ferramenta de gestão para a instituição de saúde ${ }^{3}$.

Com o advento da tecnologia da informação houve a modernização dos registros na área da saúde. O prontuário do paciente, previamente redigido de forma manuscrita em papel, passou a ser registrado de maneira eletrônica, estimulando as instituições de saúde a aderirem aos sistemas de informação para o manuseio de dados dos pacientes, tanto em países desenvolvidos, quanto nos países em desenvolvimento ${ }^{4-11}$.

Os sistemas de informação permitiram o desenvolvimento de um repositório de informação computadorizado, organizado e conciso para registro dos dados inerentes ao atendimento do indivíduo, denominado prontuário eletrônico do paciente (PEP), no qual podem ser encontrados os cuidados prestados por todos os profissionais da equipe de saúde, assim como diversas informações pertinentes a essa assistência ${ }^{1,9}$.

\begin{abstract}
Os sistemas de informação permitiram o desenvolvimento de um repositório de informação computadorizado, organizado e conciso para registro dos dados inerentes ao atendimento do indivíduo, denominado (PEP), no qual podem ser encontrados os cuidados prestados por todos os profissionais da equipe de saúde,
\end{abstract}

A utilização do PEP traz vantagens para a instituição, para os profissionais e, consequentemente, para o atendimento aos pacientes, tais como: acesso fácil, remoto e simultâneo por diversos profissionais, melhor legibilidade das anotações, segurança dos registros dos pacientes, facilidade na atualização e disponibilidade das informações clínicas do paciente, melhor continuidade no tratamento de condições crônicas, maior segurança na administração de medicamentos, assistência à pesquisa e melhor eficiência do sistema de saúde ${ }^{1,7,8}$.

Considerando os fatores que influenciam a implantação do PEP, as barreiras para o sucesso são: alto custo financeiro, demanda de tempo, conhecimento deficiente em informática por parte dos usuários, frustração com a navegação, falta de interoperabilidade com outros sistemas, necessidade de digitalizar documentos de prontuários de papel para o PEP, interfaces complexas, suporte insuficiente do fornecedor, subutilização devido à falta de conhecimento 
sobre a funcionalidade do sistema, limitações de treinamento e preocupações com a segurança das informações ${ }^{7}$.Dessa forma, entende-se que para a efetiva implantação do PEP é preciso que ocorra, primeiramente, uma mudança cultural na instituição ${ }^{3}$.

Modificar a cultura de instituições é um processo delicado, sobretudo, em unidades de terapia intensiva (UTI), ambientes permeados por protocolos e regras, que exigem dos profissionais de enfermagem apropriação para utilização de diversas tecnologias no desenvolvimento de suas atividades cotidianas. Por conseguinte, é importante explorar as percepções desses profissionais em relação às tecnologias e investigar quais percepções se relacionam com aceitação e uso, já que esses profissionais têm papel central na prestação de cuidados aos pacientes ${ }^{12}$.

O uso do PEP permeia muitas tarefas desenvolvidas pela equipe de enfermagem no decorrer de seu atendimento ao paciente crítico e problemas nesses sistemas eletrônicos ocasionam consequências não intencionais que podem prejudicar os pacientes, assim como gerar aumento da carga de trabalho para todos os profissionais da equipe de saúde envolvidos no cuidado do paciente ${ }^{13}$.

Quando a aceitação e o uso pelos profissionais são conhecidos, e os problemas relacionados ao uso são identificados, podem-se orientar projetos, redesenho, estratégias de implantação e políticas para promover aceitação e uso apropriados, melhorando a utilidade da tecnologia empregada e garantindo alta qualidade e segurança no atendimento ao paciente, sem aumento desnecessário da carga de trabalho dos profissionais ${ }^{12,13}$.

A percepção e a satisfação são constructos subjetivos, isto é, refletem uma ideia individual e são dependentes da interpretação de cada sujeito. Para medir constructos subjetivos, não basta elaborar um instrumento, é preciso testar sua validade. Um instrumento é considerado válido quando mede aquilo para o qual foi proposto medir e representa o fenômeno de interesse, sendo útil para o fim a que se destina. Uma das maneiras de se testar a validade é por meio da validade de conteúdo ${ }^{14}$.

Frente ao exposto, o objetivo deste trabalho foi avaliar a percepção sobre o uso do PEP e a satisfação dos profissionais de enfermagem de UTI, e validar um instrumento de pesquisa para este fim.

\section{Materiais e Métodos}

\section{Primeira etapa: validação de conteúdo}

O instrumento de pesquisa foi elaborado e validado pelas pesquisadoras a partir de experiência prévia, levantamento bibliográfico acerca do tema e consulta a um comitê especialistas, constituído a partir de amostra não probabilística do tipo intencional. Os especialistas foram selecionados a partir de análise do currículo lattes e verificação do preenchimento dos seguintes critérios recomendados pela literatura: domínio na área, que trabalham, estudam ou pesquisam o PEP e/ou que tenham conhecimento metodológico sobre a construção de instrumentos de pesquisa ${ }^{15,16}$. O instrumento foi avaliado em relação ao conteúdo, técnica que visa mensurar o grau em que cada item de medida é relevante e representativo de um constructo específico com um propósito particular de avaliação ${ }^{15}$. 
O grau de concordância entre os especialistas foi avaliado utilizando-se o Índice de Validade de Conteúdo (IVC), método comumente utilizado na área da saúde para quantificar o número de especialistas que concordam sobre determinados aspectos/itens do instrumento. Este método utiliza uma escala tipo Likert com pontuação de um a quatro para avaliar a relevância e clareza dos itens, sendo: 1 = não relevante ou não claro, 2 = item necessita de grande revisão para ser relevante ou pouco claro, $3=$ item necessita de pequena revisão para ser relevante ou claro, $4=$ item relevante ou muito claro ${ }^{15}$.

Adotou-se como representativo e suficiente para ser considerado validado os itens com IVC superior a 0,78 , sendo excluídos os itens que obtiveram resultados inferiores. Para obtenção do IVC calculou-se a proporção de itens que receberam a pontuação 3 ou 4 pelos especialistas, de acordo com a seguinte fórmula ${ }^{15}$ :

$$
\text { IVC }=\frac{\text { Número de respostas "3" ou "4" }}{\text { Número total de respostas }}
$$

A versão final do instrumento contemplou 40 itens, distribuídos em cinco sessões. As questões fechadas foram computadas de acordo com a frequência e a questão aberta foi categorizada em temas comuns.

\section{Segunda etapa: percepção sobre o uso do prontuário eletrônico do paciente}

Tratou-se de um estudo transversal de abordagem quantitativa, desenvolvido em um hospital público universitário localizado na cidade de São Paulo, Brasil. A amostra foi consecutiva, não-probabilística, coletada nos meses de Agosto e Setembro de 2019e constituída por 75 profissionais com formação em ensino técnico ou superior completo em enfermagem.

Os critérios de inclusão foram ser enfermeiro ou técnico de enfermagem, ter vínculo de trabalho com a instituição do estudo, trabalhar na UTI por, no mínimo, três meses, ter idade igual ou superior a 18 anos e utilizar o PEP para registro de suas atividades, rotineiramente. Foram excluídos os profissionais com menos de três meses de vínculo com a instituição para evitar viés devido a pouca familiaridade com o PEP. Não houve perda amostral.

Os dados foram armazenados em um banco elaborado no aplicativo Microsoft Excel for Windows $2010^{\circledR}$ e processados com o auxílio do software $\mathrm{R}$ versão 3.6.1. No tratamento estatístico, as variáveis categóricas foram apresentadas de forma descritiva, em tabelas e gráficos com frequências absolutas e relativas, e comparadas pelo Teste Exato de Fisher e Teste de Qui Quadrado. As variáveis numéricas foram apresentadas de forma descritiva contendo a média e o desvio padrão,e comparadas por meio do Teste de Kruskal-Wallis.

Utilizou-se a análise de conteúdo com enfoque quantitativo para analisar a questão discursiva, a qual constitui um conjunto de técnicas de análise das comunicações que envolvem procedimentos sistemáticos e objetivos de descrição do conteúdo, codificação das narrativas e exposição de sua frequência, classificando-as em categorias de acordo com os temas correlatos ${ }^{17}$.

O presente estudo foi aprovado pelo Comitê de Ética em Pesquisa do Hospital das Clínicas da Universidade de São Paulo (Protocolo n 3.527.672), estando de acordo com as normatizações 
éticas acerca das pesquisas envolvendo seres humanos previstas pela resolução 510/2016 do Conselho Nacional de Saúde ${ }^{18}$. Os indivíduos foram incluídos na pesquisa após receberem explicações sobre os objetivos e metodologia do estudo, concordarem em participar e assinarem o termo de consentimento livre e esclarecido.

\section{Resultados}

\section{Primeira etapa: validação de conteúdo}

Na primeira etapa do estudo nove profissionais da área da saúde foram convidados para compor o comitê de especialistas, por meio de correio eletrônico. Desses, sete profissionais concordaram em participar.

O Quadro 1 apresenta os dados de caracterização dos profissionais que participaram da primeira etapa. Observou-se que a média de idade entre os especialistas foi de 39 anos, sendo a maioria enfermeiros, com média de exercício da profissão de 15 anos e de experiência com PEP de 6,5 anos.

Quadro 1. Caracterização dos especialistas para validação de conteúdo. São Paulo (SP), Brasil, 2019.

\begin{tabular}{|c|c|c|c|c|c|c|}
\hline No & $\begin{array}{l}\text { Idade } \\
\text { (anos) }\end{array}$ & Profissão & $\begin{array}{l}\text { Tempo de } \\
\text { exercício da } \\
\text { profissão } \\
\text { (anos) }\end{array}$ & Formação & Área de atuação & $\begin{array}{l}\text { Tempo de } \\
\text { experiência } \\
\text { com PEP } \\
\text { (anos) }\end{array}$ \\
\hline 1 & 62 & Enfermeiro & 38 & Doutorado & Pesquisa/Docência & 3 \\
\hline 2 & 29 & Fisioterapeuta & 5 & Doutorado em andamento & Pesquisa & 7 \\
\hline 3 & 33 & Enfermeiro & 9 & Doutorado & Pesquisa/Docência & 3 \\
\hline 4 & 34 & Enfermeiro & 8 & Doutorado & Pesquisa/Docência & 0 \\
\hline 5 & 40 & Enfermeiro & 17 & Especialização/Doutorado & Assistência/Ensino & 10 \\
\hline 6 & 38 & Enfermeiro & 16 & Especialização/Doutorado & Assistência/Ensino & 10 \\
\hline 7 & 38 & Enfermeiro & 15 & Doutorado em andamento & Assistência/Ensino & 13 \\
\hline
\end{tabular}

Nota: PEP, prontuário eletrônico do paciente.

Fonte: Elaboração própria.

O instrumento original apresentava 41 itens. Após validação pelo comitê de especialistas, cinco itens foram incluídos, 18 itens sofreram modificações e seis itens foram excluídos, pois obtiveram IVC inferior a 0,78, ou seja, não eram suficientemente relevantes e claros para permanecer no instrumento. A versão final do instrumento passou a ter 40 itens, dispostos da seguinte forma: 1) caracterização dos profissionais de enfermagem da UTI (10 itens); 2) perfil e experiência em informática (três itens); 3) uso do PEP na UTI (18 itens); 4) avaliação de qualidade e segurança no uso do PEP na UTI (oito itens); 5) recomendações de melhorias (um item).

\section{Segunda etapa: percepção sobre o uso do prontuário eletrônico do paciente}

A amostra foi composta por 75 participantes, sendo 39 (52,0\%) técnicos de enfermagem e 36 $(48,0 \%)$ enfermeiros. Quanto à caracterização dos participantes, evidenciou-se predomínio do sexo feminino $(64,85,3 \%)$, com média de idade de 37,5 anos $( \pm 8,8)$, sendo a idade mínima 22 anos e a máxima 59 anos. A média para experiência profissional foi de 11,1 anos $( \pm 7,5)$, para tempo de admissão na instituição foi de 8,9 anos $( \pm 7,5)$, para tempo de experiência com uso do PEP foi de 1,9 anos $( \pm 1,6)$ e para número de horas de uso do computador foi de 6 horas $( \pm 4,1)$. 
O vínculo empregatício único foi predominante (57, 76,0\%), assim como, carga horária de 40 horas semanais $(39,52,0 \%)$.

Dentre os 36 enfermeiros, $33(91,6 \%)$ afirmaram ter uma ou mais especializações na modalidade latu sensu, sendo as mais frequentes: cardiologia $(23,69,6 \%)$, terapia intensiva $(11,33,3 \%)$ e gestão em saúde $(6,18,1 \%)$. Em relação à pós-graduação strictu sensu, apenas três $(8,3 \%)$ enfermeiros afirmaram possuir mestrado e nenhum havia cursado doutorado.

Com relação aos técnicos de enfermagem, 15 (38,4\%) afirmaram ter concluído ou estar cursando ensino superior. Os cursos citados foram: Enfermagem (11, 73,3\%), Administração (1, 6,6\%), Psicologia (1, 6,6\%), Pedagogia (1,6,6\%) e Tecnologia da Informação (1, 6,6\%). Dentre enfermeiros e técnicos de enfermagem, 61 (81,3\%) afirmaram ter curso básico de informática.

A maioria dos enfermeiros respondeu positivamente às afirmações acerca do uso do PEP: 20 (55,6\%) concordaram que a área para evolução de enfermagem é de fácil uso; 17 (48,6\%) concordaram que a área para elaboração dos diagnósticos de enfermagem é de fácil uso; 15 $(41,6 \%)$ concordaram que a área para estabelecimento de conduta de enfermagem é de fácil uso; 19 (52,8\%) concordaram que a área para prescrição de enfermagem é de fácil uso; 25 $(69,4 \%)$ concordaram que a área para anotações de enfermagem é de fácil uso (Figura 1); 36 (100\%) concordaram que a construção de uma interface que faça associação entre diagnóstico, prescrição, condutas e planos terapêuticos de forma pré-estruturada deve se tornar uma realidade; e 35 (97,2\%) concordaram que a construção de uma interface que faça associação entre diagnóstico, prescrição, condutas e planos terapêuticos de forma pré-estruturada deve se tornar uma realidade, pois diminuirá as chances de inconsistências na sistematização de enfermagem.

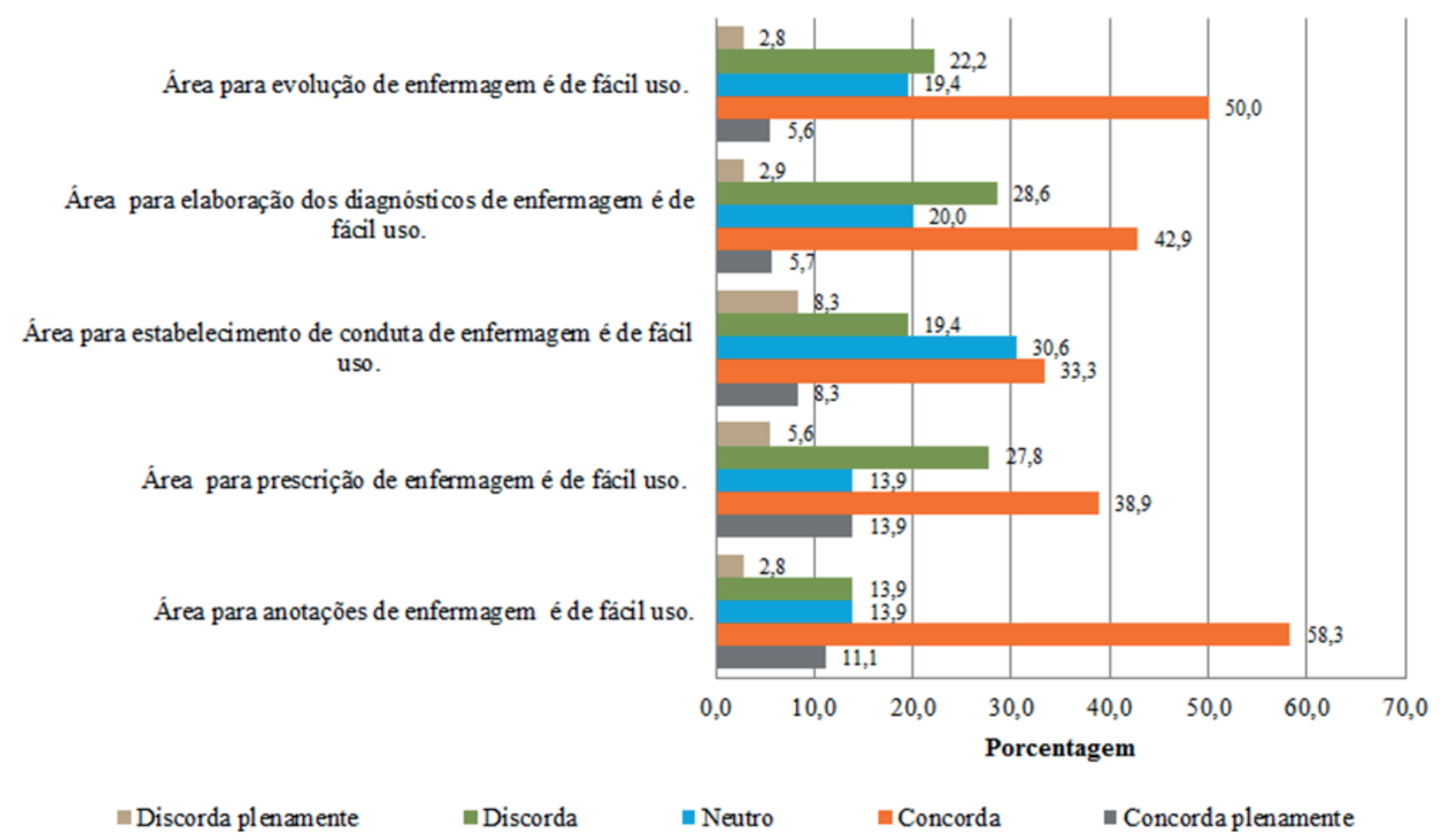

Figura 1. Percepção dos enfermeiros quanto a facilidade do uso do prontuário eletrônico do paciente. São Paulo (SP), Brasil, 2019.

Fonte: Elaboração própria. 
Dentre os técnicos de enfermagem, 15 (40,5\%) afirmaram que o limite de acesso à informação no PEP (restrição ao conteúdo visível pertinente a cada categoria profissional) facilita o trabalho, 14 (37,8\%) afirmaram que dificulta e 8 (21,6\%) ficaram neutros. Em relação à área para anotação no PEP, a maioria $(21,56,8 \%)$ afirmou que facilita o trabalho.

A Figura 2 mostra dificuldades e facilidades da equipe de enfermagem em relação ao uso do PEP, onde observa-se que 35 (47,9\%) classificaram o grau de dificuldade do PEP como fácil; $37(50,6 \%)$ consideraram a configuração do sistema boa; 54 (74,0\%) discordaram sobre haver quantidade de computadores suficientes; $52(71,2 \%)$ concordaram que possuem conhecimento suficiente sobre as funcionalidades do PEP para realizar as atribuições que lhes cabem; e 36 $(49,3 \%)$ concordaram que a educação permanente facilita a utilização do PEP..

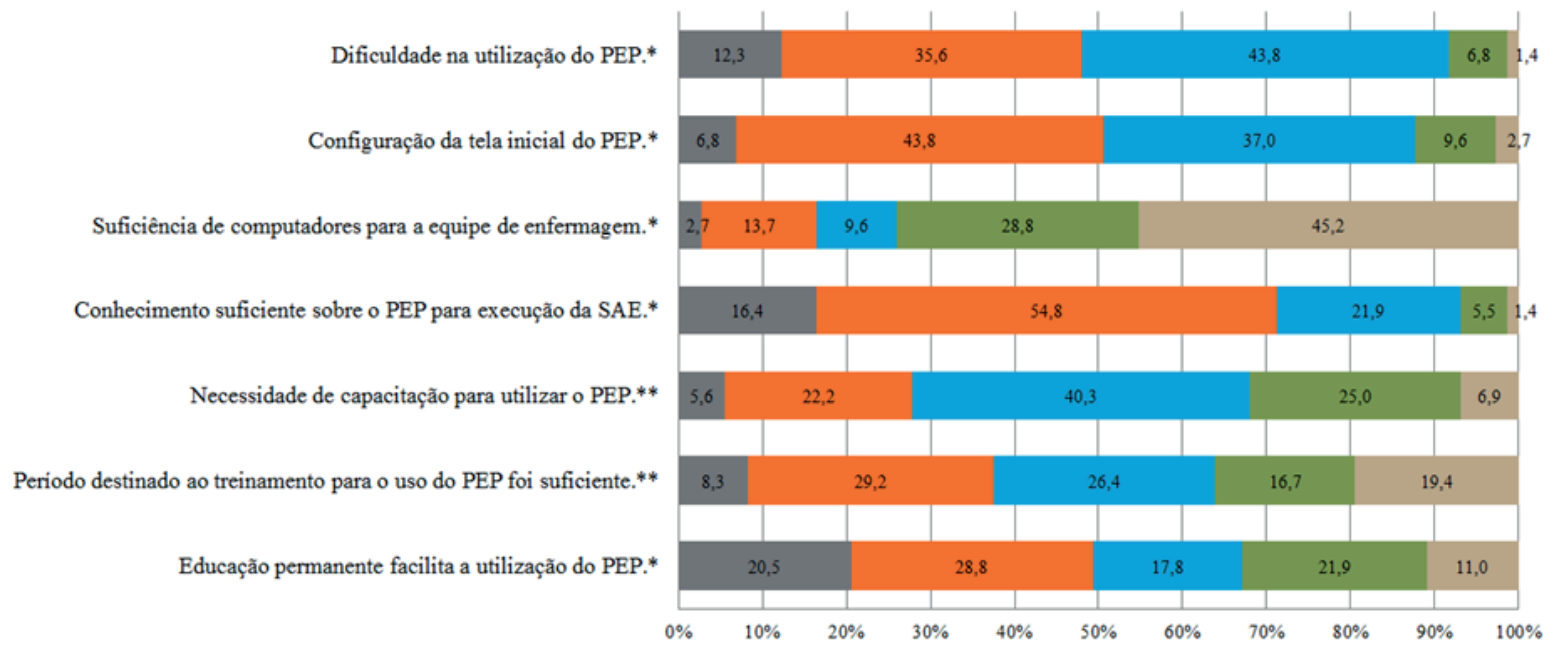

- Muito fácil / Muito boa / Concorda plenamente = Fácil / Boa / Concorda = Razoável / Razoável / Neutro = Dificil / Ruim / Discorda = Muito dificil / Muito ruim / Discorda plenamente

Figura 2. Percepção da equipe de enfermagem sobre as dificuldades e facilidades da utilização do prontuário eletrônico do paciente. São Paulo (SP), Brasil, 2019.

Nota: PEP, prontuário eletrônico do paciente; $\mathrm{SAE}$, sistematização da assistência de enfermagem;

${ }^{*}$ Considerar $\mathrm{n}=73 ;{ }^{* *}$ Considerar $\mathrm{n}=72$

Fonte: Elaboração própria.

Quanto à segurança do paciente e equipe, pode-se observar com a Figura 3 que 70 (94,6\%) participantes consideraram importante a transição do prontuário físico para o prontuário eletrônico; 42 (56,8\%)

$(60,8 \%)$ informaram maior nível de satisfação com a segurança na utilização do PEP em relação ao prontuário físico. relataram melhora na segurança do paciente, durante a assistência prestada, com a utilização do PEP; 41 (55,4\%) relataram que houve melhora na segurança da equipe de saúde com a utilização do PEP; e 45 (60,8\%) informaram maior nível de satisfação com a segurança na utilização do PEP em relação ao prontuário físico. Por outro lado, a maioria dos profissionais discordou que o limite de tempo de uma hora para checagem de medicamentos e cuidados de enfermagem possa ser uma estratégia para aumentar a segurança do paciente. 


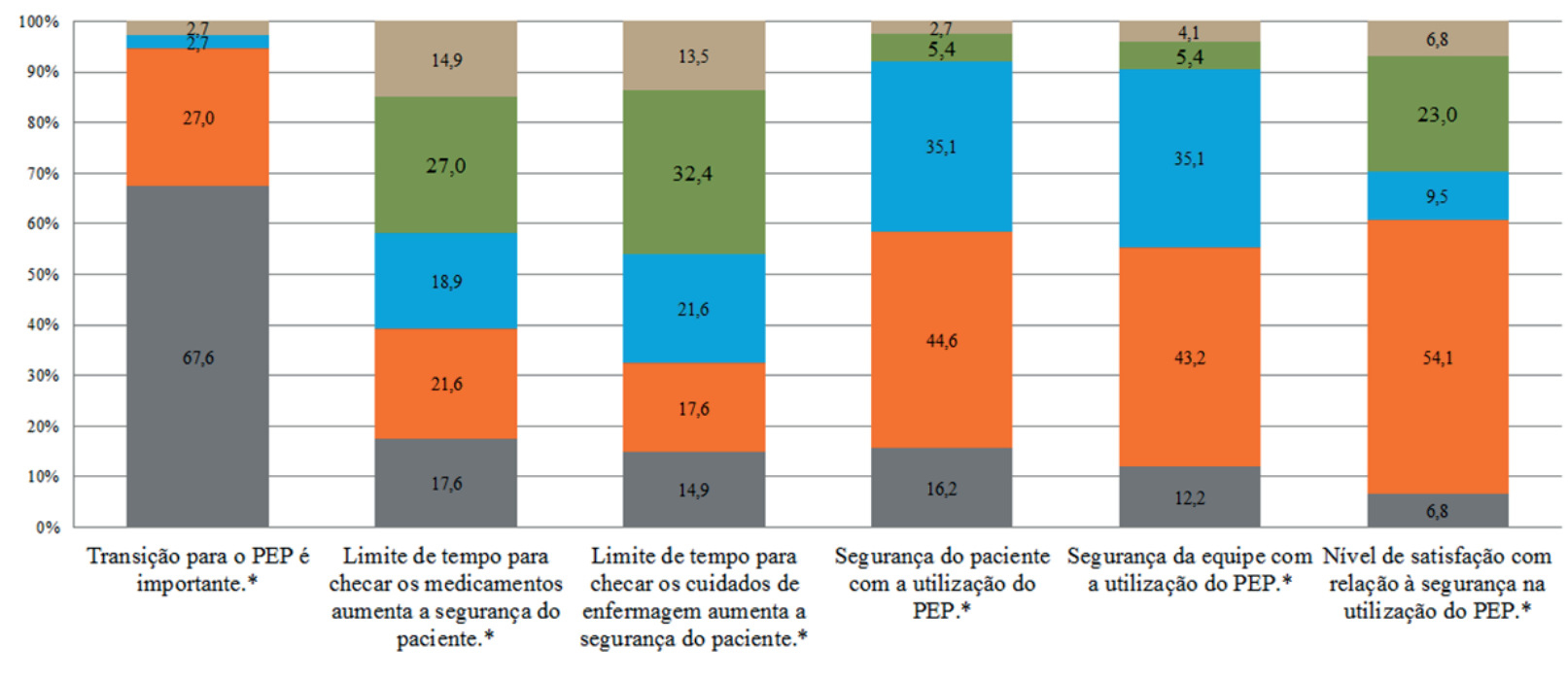

- Concorda plenamente / Muito melhor/Muito satisfeito neutro / Indiferente / Indiferente

- Concorda / Melhor / Satisfeito

- Discorda plenamente / Muito pior / Muito Insatisfeito

a Discorda / Pior / Insatisfeito

Figura 3. Percepção da equipe de enfermagem sobre a segurança com a utilização do prontuário eletrônico do paciente. São Paulo (SP), Brasil, 2019.

Nota: PEP, prontuário eletrônico do paciente; ${ }^{*}$ Considerar $\mathrm{n}=74$.

Fonte: Elaboração própria.

Em relação à qualidade, 46 (62,2\%) perceberam que a assistência multiprofissional prestada com a utilização do PEP é melhor e 35 (47,3\%) alegaram maior nível de satisfação com a qualidade na utilização do PEP em relação ao prontuário físico.

Realizaram-se associações entre as variáveis: idade, tempo de experiência profissional e tempo de admissão na instituição com o grau de dificuldade para utilização do PEP. Não houve diferenças estatisticamente significativas para estas variáveis de acordo com o teste de Kruskal-Wallis. Ao comparar a categoria profissional e o grau de dificuldade, evidenciou-se que os enfermeiros têm maior dificuldade na utilização do PEP em comparação aos técnicos de enfermagem ( $p=$ 0,013) (Tabela 1).

Quanto ao nível de satisfação em relação à segurança, o estudo mostrou que a categoria representada pelos técnicos de enfermagem demonstrou maior insatisfação com o PEP do que a categoria representada pelos enfermeiros $(p=0,005)$, assim como as mulheres demonstraram maior insatisfação em comparação aos homens $(p=0,043)$ (Tabela 1).
A categoria representada pelos técnicos de enfermagem demonstrou maior insatisfação com o PEP do que a categoria representada pelos enfermeiros $(p=0,005)$, assim como as mulheres demonstraram maior insatisfação em comparação aos homens 
Tabela 1. Nível de dificuldade e satisfação na utilização do prontuário eletrônico,de acordo com a categoria profissional, sexo e formação em informática. São Paulo (SP), Brasil, 2019.

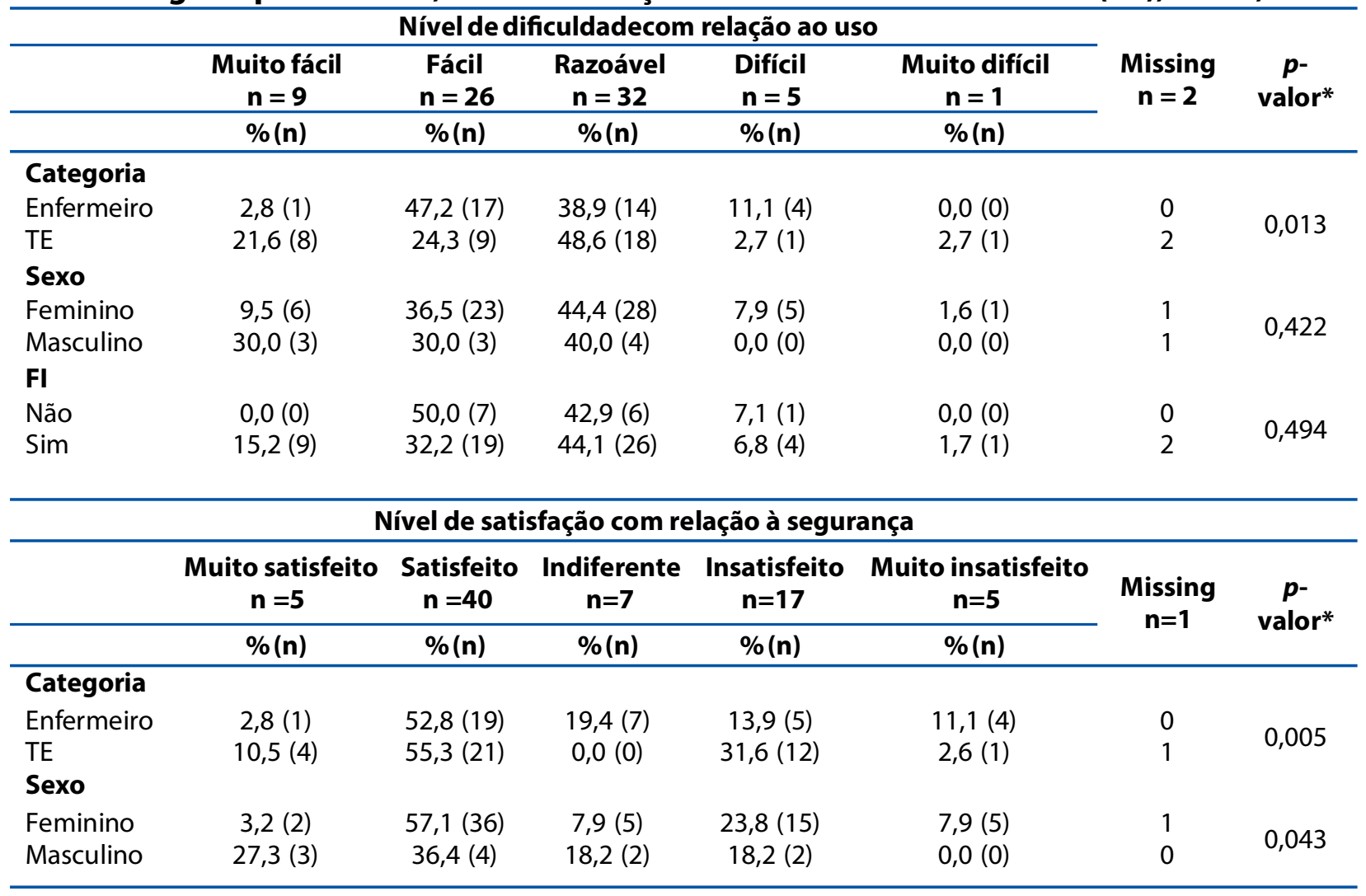

Nota: TE, técnico de enfermagem; Fl, formação em informática; *Teste Exato de Fisher.

Fonte: Elaboração própria.

Dos 75 participantes do estudo, 32(42,6\%) responderam à questão discursiva, 18 (24\%) enfermeiros e $14(18,6 \%)$ técnicos de enfermagem, sendo 28 (37,3\%) do sexo feminino e quatro $(5,3 \%)$ do sexo masculino. Esta questão permitiu ao participante responder livremente e citar as recomendações de melhorias para o uso do PEP.

Para a análise dos dados buscou-se compreender as recomendações de melhorias para o PEP, que foram decompostas em cinco categorias. A seguir serão expostas as categorias e suas respectivas frequências de aparecimento nas narrativas dos profissionais: Disponibilizar informações (anotações de enfermagem) dos pacientes para os técnicos de enfermagem (15); Aumentar prazo para checagem da prescrição médica e de enfermagem (11); Facilitar desenvolvimento da evolução e inter-relacionar as fases do processo de enfermagem (7); Facilitar mudança (aprazamento) de horário dos medicamentos (6); e Disponibilizar mais computadores (5).

\section{Discussão}

Avaliar o uso do PEP na perspectiva dos profissionais de enfermagem é uma importante forma de perceber as principais dificuldades e trabalhar de modo a saná-las, promovendo maior aceitação pela equipe e, consequentemente, garantindo melhor qualidade e segurança no atendimento ao paciente ${ }^{12,13}$.
Avaliar o uso do PEP na perspectiva dos profissionais de enfermagem é uma importante forma de perceber as principais dificuldades e trabalhar de modo a saná-las. 
Neste estudo evidenciou-se predomínio do sexo feminino, corroborando com outros estudos sobre o tema ${ }^{19-21}$, fato que pode ser justificado, historicamente, por mulheres representarem a maior parte dos trabalhadores da área da saúde, sobretudo na enfermagem ${ }^{22}$. Destaca-se que as mulheres expressaram maior insatisfação com relação à segurança no uso do PEP em comparação aos homens, assim como os técnicos de enfermagem comparados aos enfermeiros, no entanto, estes dados não foram encontrados na discussão de outros estudos.

As dificuldades encontradas pelos profissionais foram enfatizadas nas seguintes sugestões de melhorias: disponibilizar informações dos pacientes para os técnicos de enfermagem, aumentar prazo para checagem da prescrição médica e de enfermagem, facilitar desenvolvimento da evolução de enfermagem e inter-relacionar as fases do processo de enfermagem.

O número insuficiente de computadores também foi caracterizado como uma dificuldade para a utilização do PEP e atendimento ao paciente. Nessa perspectiva, estudo americano demonstrou como barreira ao uso do PEP o tempo necessário para documentar as atividades e intervenções desenvolvidas com os pacientes, além das frequentes atualizações no sistema ${ }^{23}$. Estudo brasileiro apontou que as principais dificuldades elencadas pelos profissionais de saúde referem-se à falta de equipamentos, interface complicada, falta de conhecimento em informática e pouca disponibilidade de tempo para consulta e inserção de dados dos pacientes no PEP ${ }^{20}$.

Dentre os profissionais entrevistados, a maioria discordou que o limite de tempo de uma hora para checagem de medicamentos e cuidados de enfermagem possa ser uma estratégia para aumentar a segurança do paciente. Estudos mostram que os profissionais geralmente percebem as estratégias de segurança do paciente de forma contraditória ${ }^{25}$, entretanto, faz-se necessária a análise de percepção da equipe como uma estratégia de segurança, tendo em vista estar em consonância com as metas internacionais de segurança do paciente propostas pela Organização Mundial da Saúde (OMS) ${ }^{25}$.

A quantidade de técnicos de enfermagem que relatou que o limite de acesso à informação imposta a eles facilita o trabalho equiparou-se aos que relataram que dificulta. Estudo realizado no Brasil apontou que limitar o acesso às funcionalidades do sistema caracteriza uma dificuldade do ponto de vista dos enfermeiros ${ }^{21}$.

A literatura mostra que a participação dos usuários nas etapas que precedem a implantação do sistema, bem como sua familiaridade com o mesmo e a adoção de sugestões elaboradas pelos profissionais que vivenciam na prática as mudanças contribui para torná-los parceiros e colaboradores na divulgação do PEP, e condicionam o sucesso ou o fracasso de sua implantação ${ }^{26}$. Em contrapartida, enfermeiros referem que implantar um sistema de prontuário eletrônico sem conhecer a opinião dos usuários pode dificultar ainda mais o processo ${ }^{21}$.
A participação dos usuários nas etapas que precedem a implantação do sistema, bem como sua familiaridade com o mesmo e a adoção de sugestões elaboradas pelos profissionais que vivenciam na prática as mudanças contribui para torná-los parceiros e colaboradores na divulgação do PEP, e condicionam o sucesso ou o fracasso de sua implantação ${ }^{26}$

Neste estudo, o número de pessoas que concordaram que o treinamento oferecido para utilização do PEP foi suficiente equiparou-se ao número de pessoas que discordaram. A falta de treinamento é um fator observado em outros estudos, o qual, quando associado à falta de 
habilidade em informática, pode ser responsável pela resistência ao uso do PEP20,21. Por outro lado, o desenvolvimento de estratégias que visam à capacitação dos profissionais favorece a implantação e o uso do PEP 23,26 .

A maioria dos profissionais entrevistados afirmou possuir formação em informática, dado que se assemelha ao de estudo recente realizado em município brasileiro ${ }^{27}$. Tal formação pode ter colaborado para a facilidade expressa pelos profissionais em utilizar o PEP no presente estudo. Estudo realizado com a equipe de enfermagem de um hospital terciário do Rio de Janeiro, que objetivou testar o desempenho dos profissionais após um programa de educação permanente para uso do PEP evidenciou que os enfermeiros obtiveram melhor desempenho do que os técnicos de enfermagem ${ }^{19}$. No presente estudo demonstrou-se o oposto, ou seja, os enfermeiros expressaram maior grau de dificuldade em relação aos técnicos de enfermagem. Este fato pode ser justificado pela distinção nas atribuições desenvolvidas por cada categoria, visto que a depender da função exercida, o profissional necessitará de determinado nível de domínio para uso do PEP.

Frente ao exposto, vale salientar que a educação permanente é uma importante estratégia para sanar dúvidas e minimizar dificuldades, além de capacitar os funcionários recém-contratados e qualificar rotineiramente os funcionários que se deparam com frequentes atualizações e mudanças no sistema, de modo que o PEP possa contribuir no planejamento da prática de enfermagem, facilitando o atendimento às reais necessidades do paciente ${ }^{19}$.

No que se refere às potencialidades, sabe-se que para avaliação de um sistema na perspectiva do usuário final, um dos fatores mais importantes é a interface de comunicação entre o usuário e o sistema, que deve ser de fácil aprendizagem e intuitiva ${ }^{28}$. Outras potencialidades da utilização do PEP são: qualificação da informação registrada; organização do serviço, permitindo a comunicação entre os profissionais da equipe multiprofissional; melhor planejamento e supervisão do cuidado; continuidade da assistência; e cooperação com a pesquisa ${ }^{21}$. No presente estudo, a maioria dos enfermeiros concordou que a construção de uma interface de fácil uso e que faça associação entre as etapas do processo de enfermagem é importante e deve se tornar uma realidade.

As vantagens inerentes ao uso da tecnologia da informação na área da saúde estão bem definidas na literatura, no entanto, apesar dos sistemas informatizados terem sido desenvolvidos e inseridos nessa área com o intuito de assegurar tais vantagens, e melhorar a eficiência e a produtividade da equipe multiprofissional, a garantia desse sucesso depende da própria equipe, no sentido de ter aceitação e disponibilidade para iniciar um processo de mudança29.

Na perspectiva dos profissionais de saúde atuantes em UTI, a informatização do prontuário do paciente diminui a carga de trabalho e contribui para a qualidade do atendimento neste cenário. Outras vantagens são o uso de um sistema preciso e amigável ao usuário, e a obtenção de informação clara dentro de um período de tempo razoável ${ }^{6}$.

De modo geral, a implantação e utilização adequada do PEP proporciona melhor legibilidade dos registros e da prescrição médica ${ }^{30}$, contribuindo para a segurança durante a assistência de acordo com as metas internacionais de segurança do paciente propostas pela OMS, a saber: identificação correta do paciente, comunicação efetiva entre a equipe e administração segura de medicamentos ${ }^{25}$. 
Em 2017 a OMS lançou a terceira edição do Desafio Global para Segurança do Paciente, documento que pressupõe ações em segurança do paciente em todos os países, visando minimizar os riscos relacionados aos erros de medicação. Nesse interim, o uso de tecnologias como o PEP, pode auxiliar os profissionais de saúde a garantir o uso adequado e seguro de medicamentos, principalmente dos medicamentos potencialmente perigosos. O PEP também pode contribuir para uma comunicação efetiva nos momentos de transição de cuidados do paciente, minimizando erros em decorrência de uma comunicação ineficaz ${ }^{31}$.

\section{Conclusão}

As principais dificuldades expressas pela equipe de enfermagem foram quanto ao número insuficiente de computadores, limite de acesso às informações e curto prazo para checagem das prescrições médica e de enfermagem. Os enfermeiros expressaram maior dificuldade para utilização do PEP em comparação aos técnicos de enfermagem.

O instrumento utilizado para coleta de dados foi validado em relação ao conteúdo e sua versão final foi útil para avaliar a percepção e satisfação sobre o uso do PEP em UTI pela enfermagem. O PEP foi considerado mais seguro do que o prontuário físico, fácil de utilizar e com boa configuração. A interface para desenvolver o processo de enfermagem foi apontada como uma facilidade, assim como a capacitação sobre suas funcionalidades.

Sugestões de melhorias foram apontadas pela equipe, como disponibilizar mais informações dos pacientes e computadores para uso, facilitar o aprazamento de medicamentos, aumentar o prazo para checagem de itens e facilitar a conexão entre as fases do processo de enfermagem. O estudo mostra que o sucesso na adesão de uma tecnologia depende da facilidade do uso e satisfação dos usuários. Com isso, educação permanente conforme demanda, treinamentos direcionados e investimentos em cultura de segurança são necessários para enaltecer a importância do uso correto do PEP, torná-lo uma ferramenta aliada do profissional na segurança e qualidade da assistência, além de reconhecer falhas e intervir com possíveis melhorias.

Conflito de interesse: As autoras declaram ausência de conflito de interesse.

Financiamento: Não houve financiamento.

\section{Referências}

1. Massad E, Marin HF, Azevedo Neto RS, editores. O prontuário eletrônico do paciente na assistência, informação e conhecimento médico. Available from:

http://www.sbis.org.br/biblioteca_virtual/prontuario.pdf.

2. Cofen. Conselho Federal de Enfermagem. ResoluçãoCofennº 564, de 06 de novembro de 2017. Aprova o novo Código de Ética dos Profissionais de Enfermagem. Brasília, DF, 2017.

3. Jenal S, Évora YDM. Desafio da implantação do prontuário eletrônico do paciente. J. Health Inform.2012;4 (Número Especial - SIIENF 2012):216-9.

Available from: http://www.jhi-sbis.saude.ws/ojs-jhi/index.php/jhi-sbis/article/view/253/151

4. Farias JS, Guimaraes TA, Vargas ER, Albuquerque PHM. Adoção de prontuário eletrônico do paciente em hospitais universitários de Brasil e Espanha: a percepção de profissionais de saúde. Rev. Adm. Pública. 2011;45(5):1303-26.

https://doi.org/10.1590/S0034-76122011000500004 
5. Lima AFC, Melo TO. Percepção de enfermeiros em relação à implementação da informatização da documentação clínica de enfermagem. Rev. ESC. Enferm. USP. 2012;46(1):175-183. https://doi.org/10.1590/S0080-62342012000100024

6. Fumis RRL, Costa ELV, Martins OS, Pizzo V, Souza IA, Schettino GPP. A equipe da UTI está satisfeita com o prontuário eletrônico do paciente? Um estudo transversal. Rev. Bras. Ter. Intensiva. 2014;26(1):1-6. https://doi.org/10.5935/0103-507X.20140001

7. Carpeggiani C, Macerata A, Morales MA. Electronic medical record in cardiology: a 10-year Italian experience. Rev. Assoc. Med. Bras. 2015;61(4):317-23.

https://doi.org/10.1590/1806-9282.61.04.317

8. Chang F, Gupta N. Progress in electronic medical record adoption in Canada. Can. Fam. Physician. 2015;61(12):1076-84.

9. Lahm JV, Carvalho DR. Prontuário eletrônico do paciente: avaliação de usabilidade pela equipe de enfermagem. Cogitare Enferm. 2015;20(1):38-44.

http://dx.doi.org/10.5380/ce.v20i1.36485

10.Scantlebury A, Sheard L, Watt I, Cairns P, Wright J, Adamson J. Exploring the implementation of an electronic record into a maternity unit: a qualitative study using Normalisation Process Theory. BMC Med. Inform. Decis. Mak. 2017;17(1):4.

https://doi.org/10.1186/s12911-016-0406-0

11.Colleti JJ, Andrade AB, Carvalho WB. Avaliação do uso de sistemas de prontuário eletrônico nas unidades de terapia intensiva brasileiras. Rev. Bras. Ter. Intensiva. 2018;30(3):338-46. https://doi.org/10.5935/0103-507x.20180057

12.Holden RJ, Asan O, Wozniak EM, Flynn KE, Scanlon MC. Nurses' perceptions, acceptance, and use of a novel in-room pediatric ICU technology: testing an expanded technology acceptance model. BMC Med. Inform. Decis. Mak. 2016;16(1):145.

https://doi.org/10.1186/s12911-016-0388-y

13.Lopez KD, Fahey L. Advocating for Greater Usability in Clinical Technologies: The Role of the Practicing Nurse. Crit. Care Nurs. Clin. North Am. 2018;30(2):247-57.

https://doi.org/10.1016/j.cnc.2018.02.007

14.Fayers PM, Machin D. Quality of Life. 2nd ed. London: Wiley; 2013.

15.Alexandre NMC, Coluci MZO. Validade de conteúdo nos processos de construção e adaptação de instrumentos de medidas. Ciênc. Saúde Coletiva. 2011;16(7):3061-8.

https://doi.org/10.1590/S1413-81232011000800006

16.Melo RP, Moreira RP, Fontenele FC, Aguiar ASC, Joventino ES, Carvalho EC. Critérios de seleção de experts para estudos de validação de fenômenos de enfermagem. Rev. Rene. 2011;12(2):424-31.

17.Bardin, L. Análise de Conteúdo. Lisboa (Portugal): Edições 70; 1995.

18.Brasil. Ministério da Saúde/Conselho Nacional de Saúde. Resolução n 510, de 07 de abril de 2016. Dispõe sobre as normas aplicáveis a pesquisas em Ciências Humanas e Sociais. Brasília, DF, 2016.

19.Cardoso RB, Ferreira BJ, Martins WA, Paludeto SB. Programa de educação permanente para o uso do prontuário eletrônico do paciente na enfermagem. J. Health Inform.2017;9(1):25-30.

20.Martins L, Sartor GD, Silva MP. Prontuário Eletrônico do Paciente: Adoção de novas tecnologias de acesso. J. Health Inform. 2019;11(3):67-73.

21.Gomes PAR, Farah BF, Rocha RS, Friedrich DBC, Dutra HS. Prontuário Eletrônico do Cidadão: Instrumento Para o Cuidado de Enfermagem. Rev. Fund. Care Online.2019;11(5):1226-1235. http://dx.doi.org/10.9789/2175-5361.2019.v11i5.1226-1235

22.Aquino EML, Menezes GMS, Marinho LFB. Women, Health and Labor in Brazil: Challenges for New Action. Cad. Saúde Públ.1995;11(2):281-290.

https://doi.org/10.1590/S0102-311X1995000200012

23.Burke MS, Ellis DM. Electronic Health Records: Describing Technological Stressors of Nurse Educators. Nurse Educ.2016;41(1):46-8. https://doi.org/10.1097/NNE.0000000000000196 
24.Reis GAX, Hayakawa LY, Murassaki ACY, Matsuda LM, Gabriel CS, Oliveira MLF. Implantação das estratégias de segurança do paciente: percepções de enfermeiros gestores. Texto Contexto-Enferm. 2017;26(2):e00340016. https://doi.org/10.1590/0104-07072017000340016

25.World Health Organization. The Conceptual Framework for the International Classification for Patient Safety. Final Technical Reportand Technical Annexes. 2009.

Available from: https://www.who.int/patientsafety/taxonomy/icps_full_report.pdf

26.Lima AFC, Melo TO. Percepção de enfermeiros em relação à implementação da informatização da documentação clínica de enfermagem. Rev. Esc. Enferm. USP. 2012;46(1):175-83. http://dx.doi.org/10.1590/S0080-62342012000100024

27. Barros MMO, Damasceno CKCS, Coêlho MCVS, Magalhães JM. Utilização do prontuário eletrônico do paciente pela equipe de enfermagem. Rev. Enferm. UFPE on line. 2020;14:e241496. http://dx.doi.org/10.5205/1981-8963.2020.241496

28.Rezende LCM, Santos SR, Medeiros AL. Assessment of a prototype for the Systemization of Nursing Care on a mobile device. Rev. Latino-Am. Enfermagem. 2016;24:e2714. https://doi.org/10.1590/1518-8345.0898.2714

29.Évora YDM. A enfermagem na era da informática (Editorial). Rev. Eletr. Enf. 2007;9(1):14.

30.Silva MSJ, Ramos IC, Leitão IMT. Percepção da equipe de enfermagem em relação a implantação do prontuário eletrônico: contribuições da tecnologia no cuidado hospitalar. Rev. Paul. Enferm. 2009;28(3):49-57. http://dx.doi.org/10.1590/1983-1447.2019.20180140

31.World Health Organization. Medication Without Harm - Global Patient Safety Challenge on Medication Safety. Geneva. 2017.

Available from: https://www.who.int/patientsafety/medication-safety/en/. 